Mediterránea Ser. Biol. (1985), n. ${ }^{\circ}$ 8. Págs. 17-28

\title{
LOS ODONATOS DE LA CUENCA DEL RIO GUADIAMAR (SEVILLA)
}

\author{
por \\ M. Ferreras Romero ${ }^{1}$ y A. Gallardo Mayenco2
}

\section{INTRODUCCION}

El hecho de que el Plan de Uso y Gestión del Parque Nacional de Doñana contemple la posibilidad de permitir nuevamente la entrada de las aguas del río Guadiamar a dicho Parque, confiere un particular interés al estudio de las comunidades acuáticas residentes en esta red fluvial, ya que constituyen el más fiel reflejo de la calidad de sus aguas.

Esta línea de trabajo ha sido iniciada con el estudio de la comunidad de efemerópteros presentes en el citado río y algunos de sus afluentes (GALLARDO y TOJA, en prensa).

\section{METODO}

En los años 1979 a 1981 se realizó una prospección dirigida a la recolección de todos los grupos de insectos acuáticos residentes en la cuenca del río Guadiamar. Se eligieron para ello once puntos de la red, que representan todos los tipos de medios existentes en la misma, tomándose un total de treinta y dos muestras.

(1) Departamento de Zoología. Facultad de Ciencias. Biológicas. Universidad de Córdoba.

(2) Avda. Pedro Romero, bloque 87. 41007 Sevilla. 
Para la captura de los insectos, en el caso de los Odonatos exclusivamente ninfas y exuvias, se emplearon dos mangas de caza acuática: una triangular y otra cuadrada de $30 \times 20 \mathrm{~cm}$. de lado respectivamente, ambas con malla de $0,1 \mathrm{~mm}$. de luz. Estas capturas fueron realizadas por A. Gallardo Mayenco y S. López Sánchez.

Tras examinar e identificar todas las ninfas de odonatos aparecidas en la prospección, se seleccionaron 10 muestras correspondientes a 7 estaciones, que contienen la información faunística y ecológica necesaria para la caracterización de este río en función de la comunidad de odonatos que lo ocupa. La relación numerada de estas muestras, con especificación del lugar, mediante coordenadas U.T.M., y la altitud sobre el nivel del mar, aparece en la TABLA 1.

Los datos de captura correspondientes a estas muestras, han sido tratados aplicando análisis factorial de correspondencias, con el fin de establecer la preferencia o indiferencia de las especies por los distintos biotopos de la cuenca, y estudiar posibles fenómenos de zonación a lo largo del río (VERNEAUX, 1976; GIUDICELLI et al., 1981). Se realizó un primer análisis con los datos cuantitativos originales, y después se repitió dicho tratamiento sustituyendo estos valores por clases de abundancia, correspondientes a las potencias de dos, según la escala propuesta por DUFOUR (1978a).

\section{DESCRIPCION DE LAS LOCALIDADES CONSIDERADAS EN ESTE ESTUDIO.}

Arroyo La Jarilla (muestra n. ${ }^{\circ}$ 1).-Lecho formado por rocas, gravas, arenas finas y limos. Anchura de cauce inferior a tres metros. Presenta agua durante todo el año, aunque en verano sólo existen pozas aisladas con poca profundidad (medio de carácter semipermanente). Abundante vegetación arbustiva en las orillas (Rubus sp, Nerium oleander, Cyperus sp) y Ranunculus trichophyllus en el agua.

Arroyo Aciago (muestras n. ${ }^{\circ} 2$ y 3).-Lecho constituido por rocas, cantos rodados y arenas gruesas. Anchura máxima del cauce en la zona visitada cinco metros. Curso de carácter temporal (seco durante gran parte de la época estival), muy expuesto a la insolación, por existir escasa vegetación en las orillas. En los remansos se desarrolla Ranunculus trichophyllus, especialmente abundante en los meses de primavera y principios de verano, cuando la corriente, relativamente fuerte en invierno, se hace menor o nula. 
Río Guadiamar-Castillo de las Guardas (muestras n. ${ }^{\circ} 4$ y 5).-Lecho bastante heterogéneo, formado por rocas, cantos rodados, gravas y arenas de diversos grosores. Anchura del cauce entre cinco y ocho metros. Tramo de carácter semipermanente, con fuerte corriente en la época de lluvia y sólo charcas aislàdas en el período seco. La vegetación es abundante, tanto en las orillàs como en el agua (Nerium oleander, Scirpus $\mathrm{sp}$, Juncus sp y Cyperus rotundus, Ranunculus trichophyllus y algas filamentosas).

Río Guadiamar-Las Viñas (muestra n.$^{\circ}$ 6).-Lecho constituido por arena gruesa y fina. Anchura entre ocho y diez metros. Existe agua durante todo el año, aunque en verano no hay corriente. Vegetación arbustiva en las orillas (Juncus sp, Cyperus sp, Nerium oleander) y vegetación acuática constituida fundamentalmente por Ranunculus $\mathrm{sp}$ Alisma sp y Chara sp.

Río Guadiamar-Gerena (muestra n. ${ }^{\circ}$ 7).-Localidad con características similares a las existentes en la estación anterior. Lecho de arenas y gravas. Conserva agua durante todo el año, si bien en verano sólo quedan grandes balsas aisladas entre sí (semipermanente).

Arroyo Alcárayón (muestras n.$^{\circ} 8$ y 9).-El fondo, en la zona de toma de las muestras, es fundamentalmente de limos y arenas finas, sobre los que aparecen grandes cantidades de sedimentos orgánicos en descomposición. Aunque conserva corriente durante gran parte del año, el agua es oscura, siendo casi negra en la única poza que queda durante el verano. Abundante vegetación palustre en las orillas (Typha sp, Phragmites $\mathrm{sp}$ y Scirpus $\mathrm{sp}$ ), pero escasa vegetación acuática (algas filamentosas). Anchura del cauce inferior a cinco metros.

Río Guadiamår-Aznalcázar (muestra n.$^{\circ} 10$ ).-Lecho formado por limos, arcillas, arenas, gravas y cantos rodados. Presenta agua durante todo el año, aunque en verano la corriente es mínima o nula. En este punto se registran grandes oscilaciones en el nivel de las aguas, aumentando notablemente la anchura del cauce durante la época de lluvias. La vegetación marginal no forma franjas continuas, pero puntualmente es más o menos densa (Phragmites sp, Scirpus sp y Juncus sp). El agua aparece de color verdoso a lo largo de varios meses del año, por existir en esos períodos una gran proliferación de algas. 


\section{RESULTADOS}

Las únicas referencias bibliográficas existentes sobre la fauna odonatológica del río Guadiamar y su cuenca son debidas a DUFOUR (1978b) y FERRERAS ROMERO y SOLER ANDRES (1979). El primero de estos autores recoge capturas realizadas en el río a la altura de la localidad de Sanlúcar la Mayor, en abril de 1977, citando las siguientes especies: Ischnura graellsi, Cercion lindeni, Platycnemis acutipennis, Anax parthenope, Orthetrum cancellatum, Crocothemis erythraea y Sympetrum fonscolombei. Los autores que aparecen en segundo lugar realizan capturas en julio y agosto de 1976 en el tramo más bajo del río, justo antes de entrar en la zona de las Marismas del Guadalquivir, citando las siguientes especies: Ischnura graellsi, Anax parthenope, Cronothemis erythraea y Sympetrum fonscolombei.

A continuación se presenta la relación de taxones colectados en la presente prospección, indicando la muestra o muestras a que corresponden las capturas y, entre paréntesis, el número de ejemplares (ninfas y exuvias) identificados.

Lestes viridis (Van der Linden, 1825).

Número de las muestras y ejemplares recolectados: 3(82), 5(7) y $9(28)$.

Especie con fáse ninfal de pocos meses de duración. Explota cursos de carácter temporal y algunos de carácter semipermanente, durante la primavera y principio del verano (FERRERAS ROMERO, 1982; 1984; en prensa). Durante dicho período sus poblaciones suelen estar constituidas por numerosos efectivos, especialmente en el primero de los tipos de medios citados.

Platycnemis spp.

Número de las muestras y ejemplares recolectados: $1(6), 4(8), 5(10)$, $7(1)$ y $10(6)$.

Las dos especies pertenecientes a este género presentes en Sierra Morena, $P$. acutipennis y $P$. latipes (FERRERAS ROMERO, 1982), suelen ocupar las mismas localidades, si bien el período de vuelo de la segunda está ligeramente desplazado con relación al de la primera (AGUES$\mathrm{SE}, 1960 ; 1968)$. Este hecho y la gran similitud que presentan las ninfas de ambas especies, sólo identificables cuando corresponden al último estadío, hacen aconsejable y válido, para estudios como el presente, el 
contabilizar todos los ejemplares de este género de forma conjunta. En Sierra Morena, las dos especies señaladas aparecen asociadas a cursos de agua de carácter semipermanente, como es el caso del río Guadiamar en toda su longitud.

Ischnura graellsi Rambur, 1842.

Número de las muestras y ejemplares recolectados: 1(26), 2(11), 4(4), $5(3), 6(114), 7(13), 8(19), 9(34)$ y $10(13)$.

Es el zygóptero más común en Andalucía (FERRERAS ROMERO y PUCHOL CÁBALLERO, 1984), y, en consecuencia, una de las especies más abundante y con más amplia distribución en la cuenca objeto de este estudio. Ha sido recogido en todas las estaciones consideradas y aparece, asimismo, en los trabajos anteriores referentes a este río.

Cercion lindeni Selys, 1840.

Número de las muestras y ejemplares recolectados: 1(160), 2(6), $4(39), 5(27,6(92), 7(7)$ y $10(1)$.

Especie muy abundante y difundida en las zonas alta y media de la red, resultando mucho más escasa en el tramo bajo. En Sierra Morena forma parte de la asociación característica de cursos de ríos y arroyos temporales poco mineralizados (FERRERAS ROMEŔO, 1984), hecho que se ajusta a la distribución que presenta en la cuenca del río Guadiamar, faltando únicamente en el Arroyo Alcarayón, que es el punto donde se presentan los valores más elevados de mineralización de toda la cuenca (TABLA 2).

Gomphus pulchellus Selys, 1840.

Número de las muestras y ejemplares recolectados: 1(2), 2(10), 3(2), $4(2), 5(1), 6(1), 7(4)$ y $10(1)$.

En Sierra Morena aparece formando parte de la misma asociación que la especie anterior (FERRERAS ROMERO, 1984). En el presente estudio, ambas han sido colectadas en los mismos puntos aunque el número de capturas en este caso es mucho menor.

Onychogomphus forcipatus (L., 1758).

Número de las muestras y ejemplares recolectados: 2(1), 3(3), 4(4) y $5(5)$. 
Aunque en muchos tramos de ríos de Sierra Morena suele coincidir con Gomphus pulchellus, en los cursos bajos es siempre menos frecuente. En el caso del río Guadiamar, sólo ha aparecido en uno de los arroyos de cabecera (temporal y poco mineralizado) y en el curso alto.

Paragomphus genei (Selys, 1841).

Número de la muestra y ejemplares recolectados: 5(1). .

La confirmación definitiva de la pertenencia de esta especie a la fauna española es muy reciente: TESTARD (1975) cita la captura de numerosos adultos y ninfas en una zona próxima al Parque Nacional de Doñana; las citas precedentes fueron discutidas por COMPTESART (1965). El ejemplar recogido en este trabajo es el primero capturado en Sierra Morena. En la cuenca estudiada es muy poco frecuente, existiendo, al parecer, únicamente en el curso alto del río.

Aeshna mixta (Latr., 1805).

Número de la muestra y ejemplares recolectados: 9(8).

Aunque en número relativamente considerable, sólo ha aparecido en un arroyo del tramo bajo de la cuenca, en el que se registra un elevado grado de mineralización (TABLA 2).

\section{Anax spp.}

Número de las muestras y ejemplares recolectados: 1(15), 5(2, uno A. imperator $), 6(7, \operatorname{dos} A$. imperator $), 7(3$, uno $A$. imperator $)$ y $9(2$, uno $A$. imperator).

Las ninfas de este género sólo pueden ser identificadas a nivel específico cuando son de últimos estadíos. Por este motivo, del total de ninfas recogidas, sólo cinco han podido ser determinadas a nivel infragenérico; todas ellas pertenecen a la especie $A$. imperator Leach, 1815 . Sin embargo, dado que $A$. parthenope Selys ha sido citada por DUFOUR (1978b) para el río Guadiamar, en el análisis del conjunto de las capturas será necesario considerar todos los ejemplares recolectados de este género como una única variable.

Oxygastra curtisii (Dale, 1834).

Número de la muestra y ejemplares recolectados: 5 (1 exuvia). 
Especie muy rara en Andalucía. En la cuenca estudiada parece estar limitada al curso alto del río.

Orthetrum coerulescens (Fabr., 1798).

Número de la muestra y ejemplares recolectados: 5(1).

Aunque en el presente trabajo sólo ha aparecido en tramo alto del río, en Andalucía se ha recogido en diversos tipos de cursos situados a altitudes que oscilan entre más de $700 \mathrm{~m}$. (TANAGO y VIEDMA, 1983) y el nivel del mar (TESTARD, 1975).

Crocothemis erythraea (Brullé, 1832).

Número de las muestras y ejemplares recolectados: 2(1), 4(2), 6(5), 7(3) y $9(1)$.

Siendo el anisóptero más común y difundido en Andalucía, en la cuenca estudiada aparece en la casi totalidad de las localidades consideradas. También fue capturado por los autores que con anterioridad a este estudio recogieron odonatos en otros puntos de este río.

Sympetrum striolatum (Charpentier, 1840).

Número de las muestras y ejemplares recolectados: 7(1), 8(1), y 9(37).

. Capturas anteriores realizadas en Sierra Morena ponen de manifiesto el marcado carácter oportunista de esta especie en dicha zona. Ocupa los cursos temporales, y en menor número los semipermanentes, durante la primavera y principio del verano, de forma similar a como lo hace Lestes viridis (FERRERAS ROMERO, en prensa). En la cuenca objeto de este estudio, está presente en el curso medio, no es escasa en el tramo bajo, y puede que exista en el alto, aunque no se haya detectado en esta prospección.

\section{Sympetrum fonscolombei (Selys, 1840).}

Número de las muestras y ejemplares recolectados: 4(1) y $9(1)$.

Especie ampliamente distribuida en Andalucía, ocupando una gran diversidad de biotopos; localmente puede ser muy abundante (FERRERAS ROMERO y PUCHOL CABALLERO, 1984). En este estudio se ha capturado en dos puntos de la cuenca muy distintos y distantes, apareciendo en escaso número. 
Con los datos expuestos, se ha elaborado una matriz con diez casos (muestras) y catorce variables (taxones), que ha sido tratada mediante análisis factorial de correspondencias a fin de establecer, en función de la fauna odonatológica que ocupa las distintas localidades, los diferentes tipos de medios existentes en la cuenca e investigar la existencia de una posible zonación en el río. Los porcentajes de inercia extraídos por los cinco primeros ejes son: 43,$4 ; 23,3 ; 12,3 ; 9,6$ y 6,9 .

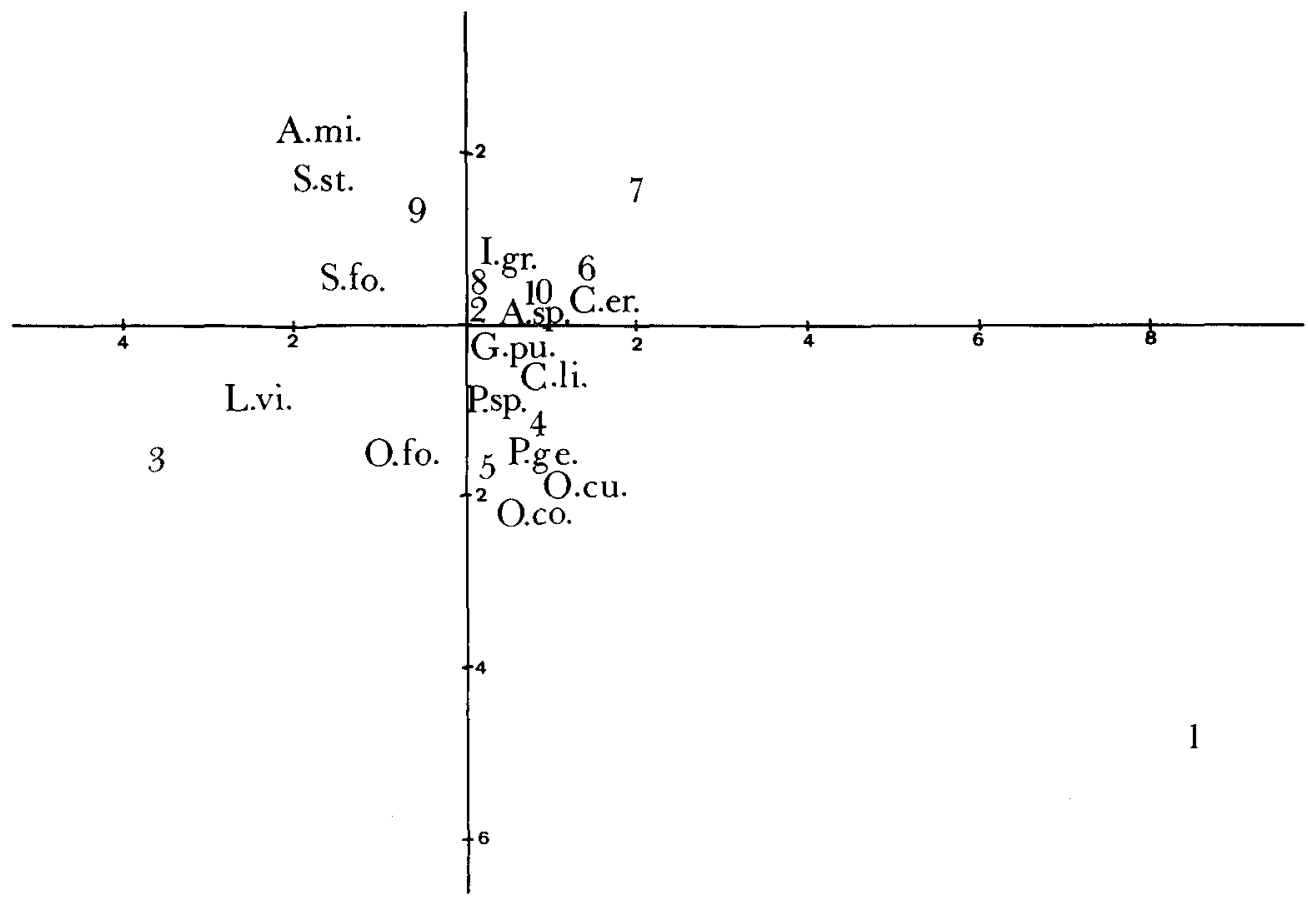

Fig. 1.-Situación de las muestras y taxones en el plano definido por los ejes I y II del análisis factorial de correspondencias realizado con datos cuantitativos.

L.vi.: Lestes viridis; P.sp.: Platycnemis spp.; I.gr.: Ischnura graellsi; C.li.: Cercion lindeni; G.pu.: Gomphus pulchellus; O.fo.: Onychogomphus forcipatus; P.ge.: Paragomphus genei; A.mi.: Aeshna mixta; A.sp.: Anax spp.; O.cu.: Oxygastra curtisii; O.co.: Orthetrum coerulescens; C.er.: Crocothemis erythraea; S.st.: Sympetrum striolatum; S.fo.: Sympetrum fonscolombei.

Al representar el resultado de este análisis en el plano definido por los dos primeros ejes (Fig. 1), se registra una cierta dispersión de los casos motivada por la disparidad de los valores de captura correspondientes a las distintas muestras, que puede suponer un efecto de ocultación producido por los taxones dominantes. Este hecho se corrige con el empleo de datos semicuantitativos, con los que se conserva la infor- 
mación necesaria para estudios de este tipo, según han demostrado VERNEAUX (1976), BORNAUD et al. (1980) y GIUDICELLI et al. (1981) entre otros. La nueva matriz ha sido elaborada utilizando para la transformación la escala de clases de abundancias, correspondientes a las potencias de dos, propuestas por DUFOUR (1978a). Se ha repetido el análisis, y los nuevos porcentajes de inercia extraídos son: 35,$4 ; 29,3 ; 12,3$; 7,5 y 6,1 . Su representación en el plano definido por los dos primeros ejes aparece en la Figura 2.

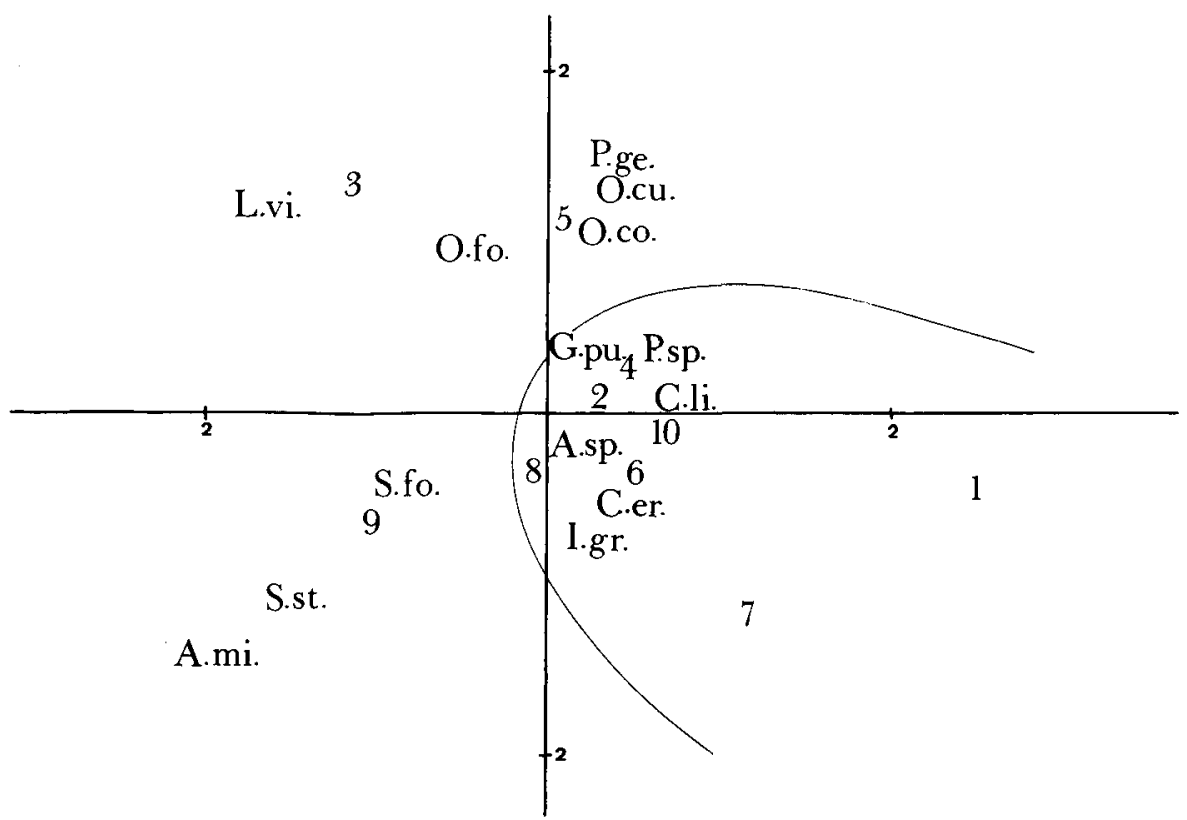

Fig. 2.-Situación de muestras y taxones en el plano definido por los ejes I y II del análisis factorial de correspondencias realizado con datos semicuantitativos.

\section{DISCUSION}

La aplicación sucesiva del análisis factorial de correspondencias a valores cuantitativos y semicuantitativos (clases de abundancia), revela la conveniencia del uso de estos últimos para la consecución del tipo de objetivos propuestos en este trabajo. Como consecuencia de ello, es precisamente en el segundo de los análisis realizados donde puede apreciarse con nitidez la existencia de diferentes asociaciones en distintos puntos de la cuenca. Aparecen tres tipos de muestras: por un lado, las 
correspondientes a meses de primavera de arroyos temporales (muestras 3 y 9), caracterizadas por la presencia, en elevado número, de una especie oportunista, Lestes viridis, y de otras, $A$. mixta y $S$. striolatum, cuya existencia puede estar condicionada por el grado de mineralización de las aguas; de otro lado, la recogida en mayo en el curso alto del río Guadiamar (muestra 5), claramente definida por la presencia de Paragomphus genei, Oxygastra curtisii y Orthetrum coerulescens; y por último, las restantes muestras, que forman un grupo caracterizado por todas las especies propias de cursos de caracter semipermanente, con corriente moderada o lenta, que en Sierra Morena son Ischnura graellsi, Cercion lindeni, Platycnemis spp, Gomphus pulchellus y Crocothemis erythraea (FERRERAS ROMERO, 1984), a las que se une Onychogomphus forcipatus en localidades del curso alto y Anax spp en gran número de puntos de la cuenca.

De lo expuesto puede concluirse que, aunque la muestra 10 (curso bajo) aparece particularmente pobre tanto en especies como en efectivos, la cuenca presenta una gran uniformidad en la mayor parte de su extensión. Unicamente la zona del curso alto, en la que han aparecido especies de gran interés, y los cursos temporales, que son explotados en primavera por elementos oportunistas, aparecen ocupados por comunidades distintas de las existentes en el resto de las localidades de la cuenca.

Por otra parte, la ausencia en esta cuenca de cursos permanentes provistos de densa vegetación en galería, impide el establecimiento y desarrollo de comunidades constituidas por especies con fases ninfales de varios años de duración, presentes en otras zonas de Sierra Morena (FERRERAS ROMERO, 1984).

Desde un punto de vista faunístico, las capturas de Paragomphus genei y Oxygastra curtisii tienen un indudable interés, ya que el número de citas ibéricas de estas especies es muy reducido. Esta es la primera cita de $P$. genei en Sierra Morena.

\section{AGRADECIMIENTOS}

Los autores desean hacer constar su gratitud a D. José M. ${ }^{a}$ Rodero Franganillo y D. ${ }^{a}$ Aurora González Domínguez, del Centro de Cálculo de la Facultad de Veterinaria de Córdoba, por la ayuda prestada en el tratamiento de los datos. 


\section{Localidad}

A. ${ }^{\circ}$ La Jarilla

A. Aciago

R. Guadiamar-

Cast. de las Guardas

R. Guadiamar-

Las Viñas

R. Guadiamar-

Gerena
A. ${ }^{\circ}$ Alcarayón
29SQB404322
R. Guadiamar-
29SQB430320
60
8 y 9
20
10

\section{Aznalcázar}

29SQB361784

300

300

2 y 3

29SQB373775

290

4 y 5

29SQB465694

200

6

29SQB484571

60

7

Tabla 1.- Situación y altitud de las localidades en que fueron tomadas las muestras consideradas en este estudio.

\begin{tabular}{|c|c|c|c|c|c|c|c|}
\hline $\begin{array}{c}\mathbf{N} .^{\circ} \\
\text { muestra }\end{array}$ & Fecha & $\begin{array}{c}\text { Tempe- } \\
\text { ratura } \\
\text { agua } \\
\left(^{\circ} \mathrm{C}\right)\end{array}$ & $\begin{array}{l}\text { Velo- } \\
\text { cidad } \\
\text { máx. } \\
(\mathbf{m} / \mathbf{s})\end{array}$ & pH & $\begin{array}{l}\text { Reserva } \\
\text { alcalina } \\
\text { (meq/1) }\end{array}$ & $\begin{array}{c}\text { Cloruros } \\
(\mathrm{mg} / 1)\end{array}$ & $\begin{array}{l}\text { Oxígeno } \\
\text { (mg/1) }\end{array}$ \\
\hline 1 & $28-7-79$ & 26,0 & 0,00 & 6,8 & 3,6 & 51,0 & 8,5 \\
\hline 2 & $12-1-79$ & 12,5 & 0,17 & 6,5 & 2,0 & 64,6 & 13,7 \\
\hline 3 & $31-5-79$ & 22,5 & 0,00 & 7,0 & 2,1 & 45,8 & 7,1 \\
\hline 4 & $27-2-79$ & 13,0 & 0,46 & 7,0 & 2,7 & 36,5 & 16,2 \\
\hline 5 & $31-5-79$ & 21,0 & 0,37 & 7,0 & 4,1 & 43,0 & 4,6 \\
\hline 6 & $21-6-81$ & 28,0 & 0,00 & 7,0 & 4,2 & 103,8 & 11,4 \\
\hline 7 & $21-6-81$ & 27,0 & 0,00 & 7,0 & 5,5 & 98,0 & 2,4 \\
\hline 8 & $9-2-79$ & 16,0 & 0,43 & 8,0 & 14,2 & 294,2 & 8,0 \\
\hline 9 & $3-4-79$ & 14,5 & 0,19 & 7,5 & 10,4 & 268,1 & 7,7 \\
\hline 10 & $3-4-79$ & 20,5 & 0,02 & 7,0 & 4,0 & 87,8 & 15,7 \\
\hline
\end{tabular}

Tabla 2.- Registros de los parámetros indicados, correspondientes a las muestras consideradas en este estudio (según Gallardo y Toja, en prensa). 


\section{BIBLIOGRAFIA}

AGUESSE, P., (1960). Notes sur l'écologie des Odonates de Provence. Année Biol., 36: 217-230.

AGUESSE, P., (1968). Les Odonates de l'Europe Occidentale, du Nord de l'Afrique et des Iles Atlantiques. Masson et Cie Ed. Paris. 258 págs.

BOURNAUD, M., G. KECK et P. RICHOUX, (1980). Les prelevements de macroinvertebres benthiques en tant que revelateurs de la physionomie d'une riviere. Annls Limnol., 16(1): 55-75.

COMPTE SART, A., (1965). Distribución, ecología y biocenosis de los Odonatos ibéricos. Publ. Inst. Biol. Aplic., 39: 33-64.

DUFOUR, C., (1978a). Etude faunistique des odonates de Suisse romande. Service des forêts et de la faune, Lausanne. 68 . II. 147 págs.

DUFOUR, C., (1978b). Odonates printaniers dans le Delta du Guadalquivir. Cahiers des Naturalistes, Bull. N.P., n.s. 32 (1976): 41-43.

FERRERAS ROMERO, M., (1982). Odonatos de Sierra Morena Central (Córdoba). Aspectos faunísticos. Bol. Asoc. esp. Entom., 5 (1981): 13-23.

FERRERAS ROMERO, M., (1984). The odonate communities associated with distinct aquatic environments of the Sierra Morena (Andalusia), Spain. Notul. Odonatol., 2 (4): 57-61.

FERRERAS ROMERO, M., (en prensa). La fauna odonatológica de la cuenca del embalse del río Bembézar (Sierra Morena) en un período de pluviometría irregular (1982-1983). Studia Oecologica.

FERRERAS ROMERO, M. y V. PUCHOL CABALLERO, (1984). Los Insectos Odonatos en Andalucía. Bases para su estudio faunístico. Ser. Publ. Univ. Córdoba, Córdoba, 160 págs.

FERRERAS ROMERO, M. y A.G. SOLER ANDRES, (1979). Odonatos de las Marismas det Bajo Guadalquivir. Aspectos faunísticos. Bol. Asoc. esp. Entom., 3: 213-218.

GALLARDO, A. y J. TOJA, (en prensa). Distribución de los efemerópteros en el río Cuadiamar (Sievilla). Actas II Congreso de Limnología (Murcia, 1983).

GIUDICELLI, J., A. DIA y P. FLEGIER, (1981). Etude hydrobiologique d'une rivière de région méditerranéenne, l'Argens (Var, France). Bijdragen tot de Dierkunde, 50 (2) (1980): 303-341.

TANAGO, M.G. y M.G. de VIEDMA, (1983). Consideraciones acerca de los Efemeroptera, Odonata y Plecoptera del Coto Nacional de las Sierras de Cazorla y Segura. Misc. Zool., 7 (1981): 53-66.

TESTARD, P., (1975). Note sur l'émergence, le sex-ratio et l'activité des adultes de Mesogomphus genei Selys, dans le Sud de l'Espagne (Anisoptera: Gomphidae). Odonatol., 4 (1): 11-26.

VERNEAUX. J., (1976). Biotypologie de l'écosysteme «eau courante». La structure biotypologique. C.R. Acad. Sc. Paris, 283 (D): 1.663-1.666. 